\title{
Bone Densitometry in Children with Chronic Renal Failure
}

A.S.Elsayed, H.R.Omar, H.T.Khater and S.E.Mohammed

Pediatrics Dept., Faculty of Medicine, Benha Univ., Benha, Egypt

E-Mail: Shymaa@gmail.com

\begin{abstract}
Interminable kidney ailment mineral and bone issue (CKD-MBD) is made out of clinical, biochemical and radiological irregularities related with CKD. The point of this examination was to survey the Bone mineral thickness in youngsters with ceaseless renal disappointment by utilizing Dual-vitality X-beam absorptiometry (DXA) which is the most broadly utilized technique to evaluate bone healthThis study included 22 kids with pediatric beginning of Chronic Renal Failure (CRF) at the Children's Dialysis Unit at Pediatric office at Benha University Hospital that were evaluated for bone mineral thickness by utilizing Dual-vitality X-beam absorptiometry (DXA). There was a critical positive connection among's BMD and PTH $(r=0.504$ and $\mathrm{P}$ esteem $=0.024)$ and huge positive relationships among's BMC and tallness No noteworthy connections among's BMD and sexual orientation, family background of CKD and Ascites.. In our investigation a greater part of patients with CKD had low degree of BMD. Moreover, lower levels of Ca, P, PTH were found in patients with strange BMD Z-scores.
\end{abstract}

\section{Introduction}

Interminable kidney ailment (CKD) is characterized as a reformist loss of renal capacity that goes on for over 3 months, and is ordered by the level of kidney harm - estimated by the degree of proteinuria - and the decrease in glomerular filtration rate(GFR). The most serious structure is end-stage renal disease.Chronic kidney infection (CKD) influences over 20million people in the USA and 752 million people worldwide [1].

People with CKD have a high pervasiveness of CKD mineral and bone infection (CKD-MBD). CKD-MBD envelops a scope of anomalies remembering confusions for mineral digestion, vascular and delicate tissue calcifications, and skeletal variations from the norm. In patients with $\mathrm{CKD}$, osteoporosis and break hazard are higher than those in the general population [2] effectsly affect personal satisfaction, and [3] increment the danger of mortality.

Osteoporosis is characterized by the (National Institutes of Health NIH Consensus Development Panel on Osteoporosis) as a skeletal issue portrayed by undermined bone quality inclining an individual to an expanded danger of crack [4].

Bone quality is dictated by bone amount and bone quality. Bone Mineral Density(BMD) can be estimated utilizing double vitality X-beam absorptiometry(DEXA), which is brisk, non-intrusive and clear to perform and the radiation presentation is exceptionally low.

Low BMD has been portrayed in both highturnover related with hyperparathyroidism and in low-turnover related with lower (frequently typical) Para Thyroid Hormone levels(PTH) [4].

Grown-up information are various and relationship between intensifying Chronic Renal Failure(CRF) or potentially raised PTH levels and diminishes in BMD have been demonstrated [5].

Pediatric information examining BMD utilizing DEXA imaging contemplates show diminished [5] and others typical BMD [6].
These irregularities are perhaps because of little patient numbers and changing patient qualities just as contrasting helpful administration procedures influencing bone turnover: calcitrol treatment utilized in few kids on peritoneal dialysis with optional hyperparathyroidism impacted BMD well, however contrasts among oral and intravenous organization were noted [7] Studies examining the impacts of development hormone treatment upon BMD are discordant $[8,9]$.

The point of this investigation was to evaluate Bone mineral thickness in youngsters with constant renal disappointment by utilizing Dual-vitality $\mathrm{X}$ beam absorptiometry (DXA) which is the most broadly utilized technique to survey bone wellbeing.

\section{Patient and method}

This examination was a cross sectional investigation, which was done on 20 cases with interminable renal disappointment on hemodialysis (at the Children's Dialysis Unit at Pediatric division, Benha University Hospital,) in the period from august 2019 till may 2020.

\subsection{Ethical contemplations}

Moral authorization for the examination was acquired from the guardians who were completely educated pretty much all investigation methodology and their assent was gotten preceding the youngsters enlistment in the examination. This investigation was affirmed by the moral board of the workforce of medication, Benha University .

\section{Incorporation models}

20 instance of youngsters with ceaseless renal disappointment who was surveyed bone mineral thickness by utilizing Dual-vitality X-beam absorptiometry (DXA)

- Both genders were incorporated.

- Laboratory models for constant renal disappointment.

- Avoidance measures:

- No avoidance measures. 
- All patients were exposed to finish history with weight on sex, age, period of beginning, length, home, family background of CRF, sort of treatment, its term.

- General assessment.

- Anthropometric estimations: including weight, stature and Z-score were recorded. Weight list (BMI), BMI z-scores will be determined utilizing the (WHO) Child Growth Standards and Growth Reference information with the WHO anthropometric adding machine, AnthroPlus v.1.0.4.

\section{Nearby assessment}

- Cardiovascular assessment: With Stress on Sign of pericardial radiation.

- Pulmonary assessment: With Stress on Sign of chest contamination and pleural emanation.

- Abdominal assessment: for ascitis and oedema of front stomach divider.

- Genitourinary framework assessme

- nt: for indication of oedema with weight on scrotal oedema.

\section{Laboratory investigations \\ Blood sample}

$4 \mathrm{ml}$ of blood sample were taken in plain test tubes were allowed to clot for $30 \mathrm{~min}$. at room temperature and centrifuged for $15 \mathrm{~min}$. at $1000 / \mathrm{min}$. the serum was removed, aliquoted and stored at $<20{ }^{\circ} \mathrm{c}$ until assayed, the separated serum was used for the following assays :

- Serum creatinine and blood urea using biosystem A15 autoanalyzer (Biosystems S.A., Barcelona , Spain )

- Serum phosphorous (P),

- Serum calcium (total and ionized; $\mathrm{Ca}$ ),

- Serum alkaline phosphatase (ALP).

- All serum calcium, serum phosphorus and alkaline phosphatase are done by Biochemistry analyzer (Biosystem) A15 autoanalyzer. lot no of (calcium, phosphorus and alkaline phosphatase) is (16005,15007 and15-0841).

- Serum parathyroid hormone (PTH), Serum intact parathyroid hormone using a direct label, two-site ELISA assay intended for the quantitative determination of PTH in serum ( Biomerica, USA; normal range: 10-65 pg/ml) lot no (2329), company name (Diasorin) and instrument name (Diasorin Liaison) PTH 4-2016 / W0886200.

- Serum vitamin D.

- BMD was measured at the lumbar spinal region (L2-L4) using dual-energy X-ray absorptiometry (DXA) (Challenger Envision osteodensitometer,
DMS, England). BMD was classified according to (5), on the basis of BMD Z-score. Scores was calculated from the following equation: Z-score $=$ (BMD [ $/ \mathrm{cm} 3]$ of the patient - BMD predicted for age and sex/SD for BMD [age, sex, and height matched]).

A patient was considered osteopenic if the Zscore was $<-1.0$. If the Z-score was $\leq-2.5$, the patient was classified as having severe osteopenia (osteoporosis).

\section{Dexa Scan}

DEXA scan is a special type of X-ray that measures bone mineral density (BMD), DEXA stands for "dual energy X-ray absorptiometry", This type of scan may also be called (DXA scan, bone density scan, bone densitometry scan)

DEXA scans are often used to diagnose or assess someone's risk of osteoporosis, a condition that weakens bones and makes them more likely to break.

\section{Results}

Mean age of study population was 15 years with standard deviation of 3 years. As regard gender, $55.0 \%$ were females and $45.0 \%$ were males

Mean onset of the disease was 9 years with standard deviation of 3 years. $53.0 \%$ showed family history of CKD. $10.0 \%$ showed family history of osteoporosis. Mean height was $130 \mathrm{~cm}$ and mean weigh was $36.0 \mathrm{~kg}$. Mean SBP and DBP were 115 and 69 respectively. $90.0 \%$ of patients showed edema, $35.0 \%$ showed ascites and $85.0 \%$ showed bone-ache.

Mean urea \& creatinine were 124 and 6.7 respectively. Mean serum ca \& PO4 were 4.5 and 2.9 respectively. As regard $\mathrm{PTH}$, median was 89.4. Mean ALP was 302 . Mean $Z$ score was -3 with standard deviation of 1.5

Mean BMI was 21.14 with standard deviation of 4.85. Mean BMD was 0.498 with standard deviation of 0.185 . Mean BMC was 26.37 with standard deviation of 12.88

There was a significant positive correlation between BMD and PTH $(r=0.504 \&$ P value $=$ 0.024)

There were no significant correlations between BMD and other parameters

There were significant positive correlations between BMC and height $(\mathrm{r}=0.739 \& \mathrm{P}$ value $<0.001)$, weight $(r=0.751 \& P$ value $<0.001)$ and creatinine $(r=0.625 \& \mathrm{P}$ value $=0.003)$

There were no significant differences in BMD as regard gender, family history of CKD and Ascites. $\mathrm{P}$ values were 0.37 . 0.485 and 0.097 respectively. 


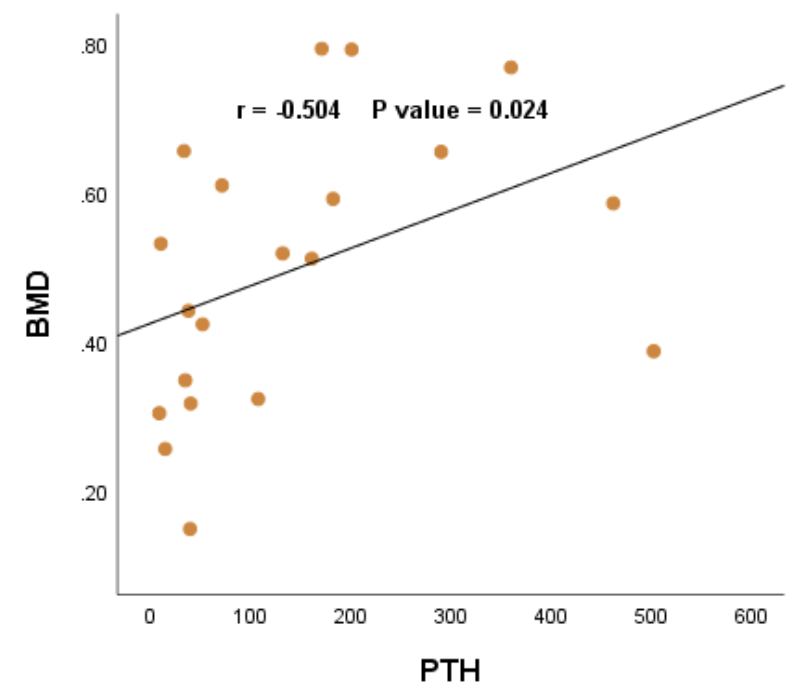

Fig (1) Correlation between BMD and PTH

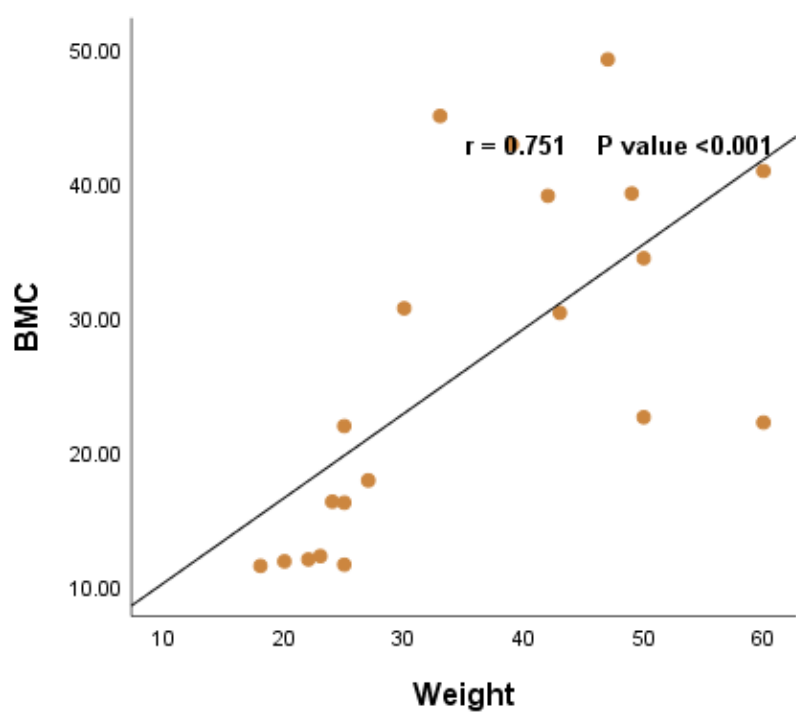

Fig (3) Correlation between BMC and weight

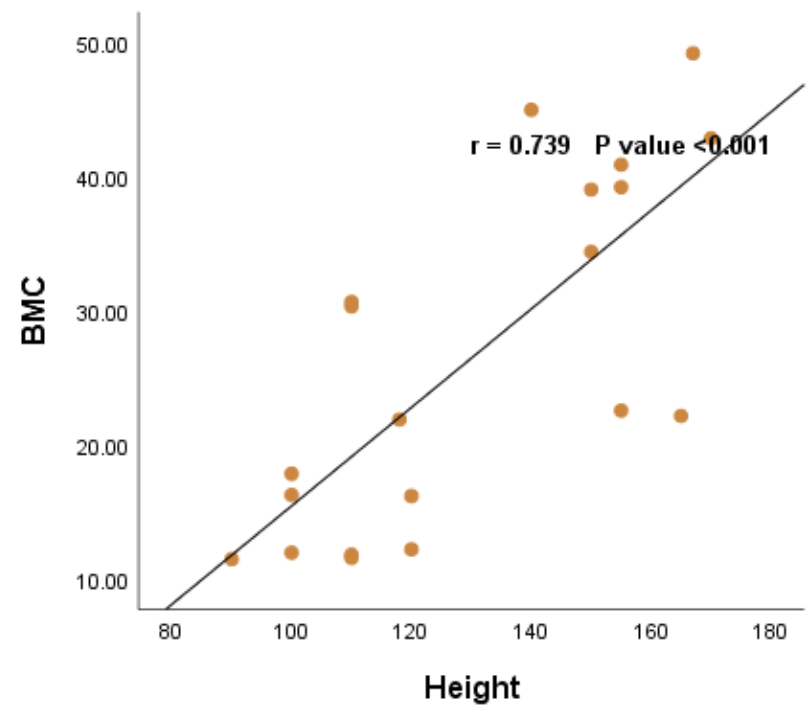

Fig (2) Correlation between BMC and height.

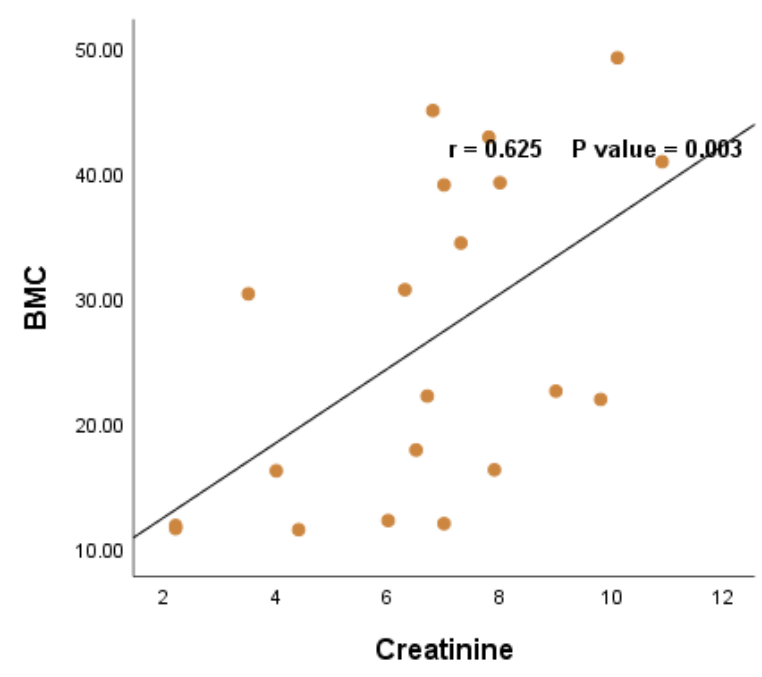

Fig (4) Correlation between BMC and creatinine.

Table (1) Bone mineral density as regard different parameters.

\begin{tabular}{lcccc}
\hline & & \multicolumn{2}{c}{ BMD } & \\
\cline { 3 - 5 } Gender & Males & 0.547 & 0.17 & 0.37 \\
& Females & 0.457 & 0.194 & \\
\multirow{2}{*}{ Family history of CKD } & Yes & 0.454 & 0.207 & 0.485 \\
& No & 0.521 & 0.176 & \\
\multirow{2}{*}{ Ascites } & & & & \\
& Yes & 0.402 & 0.124 & 0.097 \\
& No & 0.549 & 0.195 & \\
\hline
\end{tabular}

Mann Whitney U test was used. 


\section{Discussion}

The condition known as interminable kidney infection mineral and bone issue (CKD-MBD) is made out of clinical, biochemical and radiological irregularities related with $\mathrm{CKD}$, that is showed by possibly one or a blend of the accompanying variables: anomalies of calcium, phosphorus, parathyroid hormone (PTH), or nutrient D digestion, variations from the norm in bone histology, direct development, or quality, and vascular or other delicate tissue calcification [10].

Bone mineral thickness (BMD) and bone turnover are the most significant components in the arrangement of patients with CKD. It has been accounted for controlling of biochemical boundaries of CKD-MBD (e.g., serum calcium, phosphorus, and PTH levels) is so significant in these patients. Since flagging instruments between bone, kidney, and parathyroid organs, modifications in kidney capacity can provoke to changes in serum biochemical qualities [11].

Some clear and cross-sectional investigations have been accounted for about bone mass in CKD youngsters, just a couple of studies incorporate have been accounted for markers of bone turnover and the bone mass estimations. Then again, be that as it may, there are the various examinations on mineral and bone digestion in hemodialysis (HD) patients matured somewhere in the range of 50 and 80 years. An absence of studies in youthful HD patients are existed [12].

The point of this examination was to survey the Bone mineral thickness in youngsters with constant renal disappointment by utilizing Dual-vitality Xbeam absorptiometry (DXA) which is the most broadly utilized technique to evaluate bone wellbeing.

This investigation included 22 youngsters with pediatric beginning of Chronic Renal Failure (CRF) at the Children's Dialysis Unit at Pediatric office at Benha University Hospital that were surveyed for bone mineral thickness by utilizing Dual-vitality Xbeam absorptiometry (DXA).

The mean time of study populace was 15 years with standard deviation of 3 years, among them, $55.0 \%$ were females and $45.0 \%$ were guys. This coordinated the investigation by [12] that was included 27 patients with CKD, comprising 9 guys (33\%) and 18 females $(67 \%)$ with the period of 14.9 \pm 4.5 years.

Mean beginning of the ailment was 9 years with standard deviation of 3 years. 53.0\% demonstrated family background of CKD. $10.0 \%$ demonstrated family background of osteoporosis. Mean tallness was $130 \mathrm{~cm}$ and mean weigh was $36.0 \mathrm{~kg}$. Mean SBP and DBP were 115 and 69 individually. $90.0 \%$ of patients demonstrated edema, $35.0 \%$ indicated ascites and $85.0 \%$ indicated bone-throb.

It is notable that serum ALP can be utilized as a biochemical marker of high-turnover bone malady.
Serum ALP esteems are reasonable markers of osteoblastic action in youngsters with CKD. Osteoblasts typically express a lot of the bone isoenzyme of ALP, and raised serum levels relate with expanded bone development, elevated levels of serum PTH, and development hormone treatment. High mountain is available in the liver and raised serum levels of absolute ALP may not generally demonstrate expanded bone turnover [11].

Mean urea and creatinine were 124 and 6.7 separately. Mean serum ca and PO4 were 4.5 and 2.9 individually. As respect PTH, middle was 89.4. Mean ALP was 302. Mean Z score was - 3 with standard deviation of 1.5.

In [12] study, nine patients (33\%) had unusual phosphate level. Despite the fact that in the beginning phases of CKD, raised measures of circling PTH may bring about ordinary or low serum phosphate levels, in cutting edge stages, diminished glomerular filtration rate limits phosphorus discharge and lower $\mathrm{Ca}, \mathrm{P}, \mathrm{PTH}, 25(\mathrm{OH}) \mathrm{D}$ levels were found in patients with strange BMD Z-scores. In [13] study, lower calcium, 25(OH) D and higher PTH and $1,25(\mathrm{OH})_{2} \mathrm{D}$ were freely connected with lower cortical volumetric BMD Z-scores at standard.

In our investigation, the mean BMI was 21.14 with standard deviation of 4.85 , the mean BMD was 0.498 with standard deviation of 0.185 and the mean BMC was 26.37 with standard deviation of 12.88 .

There was a critical positive connection among's BMD and PTH $(r=0.504$ and $\mathrm{P}$ esteem $=0.024)$ and noteworthy positive relationships among's BMC and tallness $(\mathrm{r}=0.739$ and $\mathrm{P}$ esteem $<0.001)$, weight $(\mathrm{r}=$ 0.751 and $P$ esteem $<0.001)$ and creatinine $(r=0.625$ and $\mathrm{P}$ esteem $=0.003$ ).

In [12] study, the mean degree of PTH was emphatically associated with Z-scores $(r=0.43$, $\mathrm{P}=0.06$ for complete body $\mathrm{Z}$-score; $\mathrm{r}=0.41, \mathrm{P}=0.08$ for lumbar spine $\mathrm{Z}$-score; and $\mathrm{r}=0.45, \mathrm{P}=0.05$ for femoral neck Z-score) which bolsters our outcomes. In (14) study, among CKD members, PTH levels were emphatically connected with trabecular BMD Z-score $(\mathrm{P}<0.01)$.

Then again, [15] in his investigation indicated no measurably noteworthy relationship was seen among BMD and biochemical markers of bone turnover, while in the examination, more significant levels of PTH and biomarkers of bone arrangement (boneexplicit ALP) were related with lower cortical BMD Z-scores $(\mathrm{P} \leq 0.02)$. In [16] study, $11 \mathrm{CKD}$ patients (73\%) had expanded PTH levels at benchmark and most kids had ordinary levels nutrient $\mathrm{D}$ and it was not discovered any relationship between's Z-scores and the seriousness of CKD.

Our examination uncovered no huge relationships among's BMD and sexual orientation, family background of CKD and Ascites [12] found a positive connections between's the mean degree of ALP and lumbar spine $\mathrm{Z}$-score $(\mathrm{r}=0.46, \mathrm{P}=0.04)$ 
dissimilar to our outcomes might be because of the little example size.

\section{Conclusion}

In our study a majority of patients with CKD had low level of BMD. In addition, lower levels of $\mathrm{Ca}, \mathrm{P}$, PTH were found in patients with abnormal BMD Zscores.

\section{References}

[1] B.Bikbov, N.Perico , G.Remuzzi. on behalf of the GBDGDEG1. Disparities in chronic kidney disease prevalence among males and females in 195 countries: analysis of the Global Burden of Disease 2016 Study. Nephron, Vol. 2018, PP.16,2018 .

[2] AM. Alem, DJ. Sherrard, D.L. Gillen. Increased risk of hip mfracture among patients with endstage renal disease. Kidney Int, Vol.9_58, PP.396,2000.

[3] B.Bikbov, N.Perico , G.Remuzzi. on behalf of the GBDGDEG1. Disparities in chronic kidney disease prevalence among males and females in 195 countries: analysis of the Global Burden of Disease 2016 Study. Nephron, Vol. 2018, PP.16,2018 .

[4] AM. Alem, DJ. Sherrard , D.L. Gillen. Increased risk of hip mfracture among patients with endstage renal disease. Kidney Int, Vol.9_58, PP.396,2000.

[5] S.M. Kim, J. Long, M.Montez-Rath. Hip fracture in patients with non-dialysis-requiring chronic kidney disease. J Bone Miner Res Off J Am Soc Bone Miner Res, Vol. 2016, PP.31.9_1803,2016.

[6] H.C. Schober, Z.H. Han , A.J. Foldes, Mineralized bone loss at different sites in dialysis patients: implications for prevention. J Am Soc Nephrol,1998.

[7] BakrAM, Bone mineral density and bone turnover markers in children with chronic renal failure. Pediatr Nephrol vol.19, PP.1390-1393,2004.

[8] S.F.Ahmed, S. Russell , R. Rashid, Bone mineral content, corrected for height or bone area, measured by DXA is not reduced in children with chronic renal disease or in hypoparathyroidism. Pediatr Nephrol vol. 1472_20, PP.1466, 2005.

[9] E.Baskin, S. Ozen , M. Karcaaltincaba, Beneficial role of intravenous calcitriol on bone mineral density in children with severe secondary hyperparathyroidism. Int Urol Nephrol, Vol.36, PP.113-118,2004.

[10] I .van der Sluis, A.M. Boot , J. Nauta, Bone density and body composition in chronic renal failure: effects of growth hormone treatment. Pediatr Nephrol, Vol.15, PP.221-228,2000.

[11] MVan Dyck, A. Gyssels , W. Proesmans, Growth hormone treatment enhances bone mineralisation in children with chronic renal failure. Eur J Pediatr, Vol. 160,pp .359-363,2001.

[12] B.Waziri, R. Duarte, S.Naicker Chronic Kidney Disease-Mineral and Bone Disorder (CKD-MBD): Current Perspectives. Int J Nephrol Renovasc Dis, Vol.12, PP.263-276. Published Dec 24, 2019.

[13] N.Hajizadeh, M. Mehrkash, D. Fahimi, M. Qorbani, N. Shafa. Association of bone mineral density with biochemical markers of bone turnover in hemodialysis children. J Renal Inj Prev. 2016, Vol.5(4), PP.174-178. Published Jul 27,2016.

[14] H.S.Jørgensen, , S.Winther, , M.Bøttcher, Bone turnover markers are associated with bone density, but not with fracture in end stage kidney disease

[15] a cross-sectional study. BMC Nephrol 18, 2842017.

[16] M.R.Denburg, A.K. Tsampalieros, I.H. de Boer, J. Shults, HJ. Kalkwarf, Mineral metabolism and cortical volumetric bone mineral density in childhood chronic kidney disease. J Clin Endocrinol Metab, Vol.98, PP.1930-8,2013.

[17] R.J. Wetzsteon, H.J. Kalkwarf, J. Shults, B.S. Zemel, Volumetric bone mineral density and bone structure in childhood chronic kidney disease. J Bone Miner Res, Vol.26, PP.223544,2011.

[18] D,Sit, A.K. Kadiroglu, H. Kayabasi, A.E. Atay, Relationship between bone mineral density and biochemical markers of bone turnover in hemodialysis patients. Adv Ther, Vol.24, PP.98795,2007

[19] D. Swolin Eide, S. Hansson, P. Magnusson. Children with chronic kidney disease: a 3year prospective study of growth, bone mass and bone turnover. Acta Paediatrica, Vol.98, PP.367373,2009 . 